\title{
Surface-enhanced Raman Spectroscopy of 2D Organic Semiconductor \\ Crystals
}

Dmitry R. Maslennikova,a,c, Andrey Yu. Sosoreva,b,c, Roman S. Fedorenko ${ }^{a, b}$, Yuriy N. Luponosov ${ }^{b, d}$, Sergey A. Ponomarenko ${ }^{\mathrm{b}, \mathrm{d}}$, Vladimir V. Bruevich ${ }^{\mathrm{a}, \mathrm{b}, \mathrm{c} *}$

a Faculty of Physics and International Laser Center, Lomonosov Moscow State University, Leninskie Gory 1/62, Moscow 119991, Russia

${ }^{b}$ Enikolopov Institute of Synthetic Polymeric Materials, Russian Academy of Science, Profsoyuznaya 70, Moscow 117393, Russia

${ }^{c}$ Institute of Spectroscopy, Russian Academy of Sciences, Fizicheskaya 5, Troitsk, Moscow 108840, Russia

d Department of Chemistry, Lomonosov Moscow State University, Leninskie Gory 1/3, Moscow 119991, Russia

\section{Supporting Information}

\section{S1. Maximizing surface enhancement factor}

We studied surface enhancement factor (SEF) dependency on gold morphology controlled by gold thickness. For this, we prepared a sample with gradual varying gold thickness by placing a Si substrate at an angle to the evaporator. During evaporation the deposition rate was held close to $1 \AA$ /s. Then gold-covered substrate was covered by Langmuir-Blodgett (LB) monolayer ${ }^{1}$ for a uniform coverage along the entire length of the sample. For LB film deposition we used D2-Und-PTTP-TMS molecule represented below (Fig. S1). After SERS spectra were probed along the sample (Fig. S2, a) and signal-to-noise ratio was derived in dependence of gold thickness.

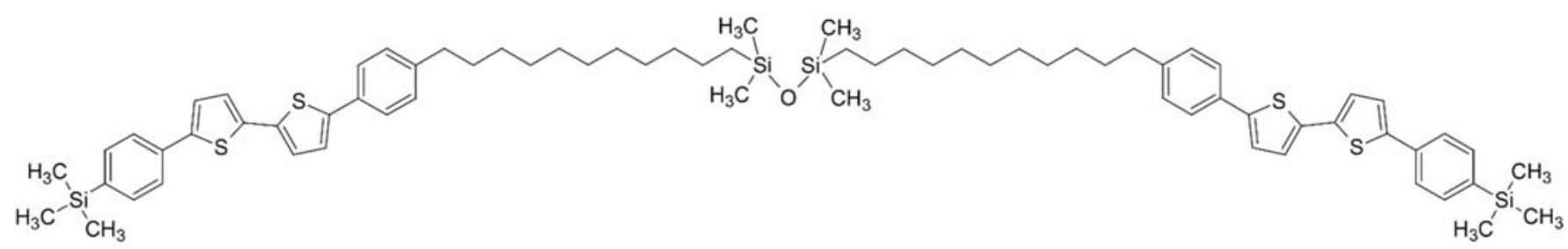

Fig. S1. Langmuir-Blodgett molecule 1,3-bis[11-(4-\{5'-[4-(trimethylsilyl)phenyl]-2,2'-bithien-5yl\}phenyl)undecyl]-1,1,3,3-tetramethyldisiloxane (D2-Und-PTTP-TMS) used for gold optimization.

Fig. S2 (b) clearly illustrates that signal-to-noise ratio has a maximum at approximately $22 \mathrm{~nm}$ of gold thickness. Hence this, we took 20-25 nm as an allowable range for preparation of SERS substrates. Gold film morphology for $24 \mathrm{~nm}$ thick gold is shown in Fig. S3. 

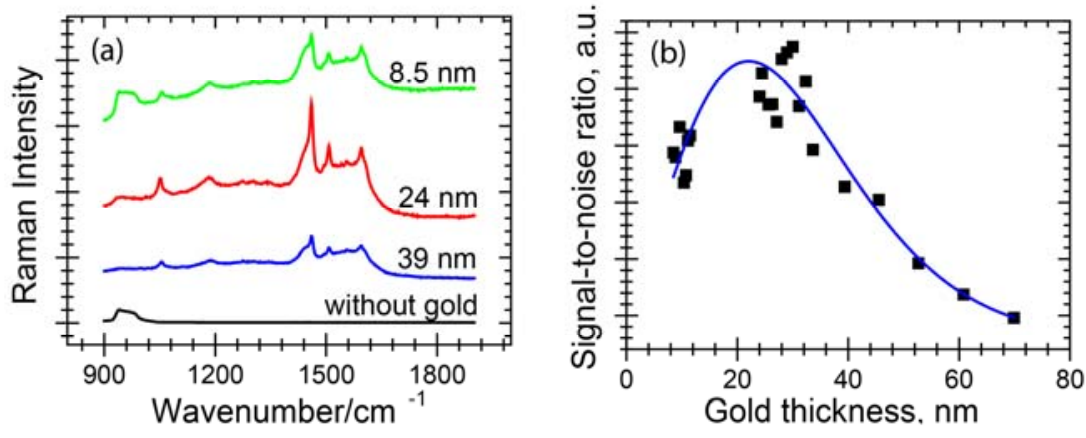

Fig. S2. (a) SERS spectra of D2-Und-PTTP-TMS for different thickness of gold in comparison with nonenhanced one. We used $10.9 \mathrm{~mW}$ laser power; 100 accumulations for enhanced spectra and 20 nonenhanced one. (b) SERS signal-to-noise dependency on the gold thickness; blue line is a guide for an eye. Thickness was measured by atomic force microscopy (AFM) in semi-contact regime.

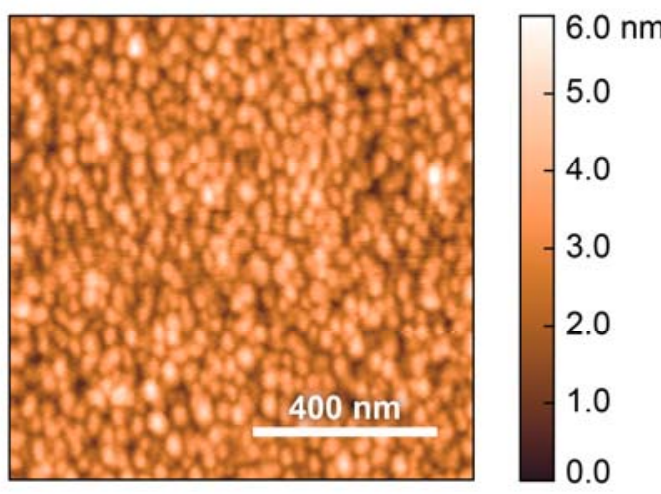

Fig. S3. AFM semi-contact scan of $24 \mathrm{~nm}$ gold. Surface roughness is $0.75 \mathrm{~nm}$ (RMS).

\section{S2. Raman anisotropy modelling}

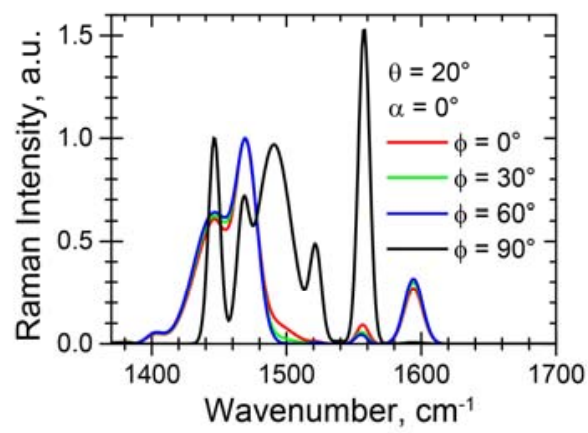

Fig. S4. Calculated anisotropy of Raman spectra assuming one molecule per a unit cell for domains with various in-plane orientations. We used DH-TTPTT calculated Raman tensor and $\theta=20^{\circ}$ simulating the inclination of DD-TTPTT molecules in the monolayer crystal. ${ }^{2}$ The spectra presented here are normalized to $1470 \mathrm{~cm}^{-1}$ peak and plotted assuming Gaussian peaks with widths taken from the experiment. For more details see the main text. 

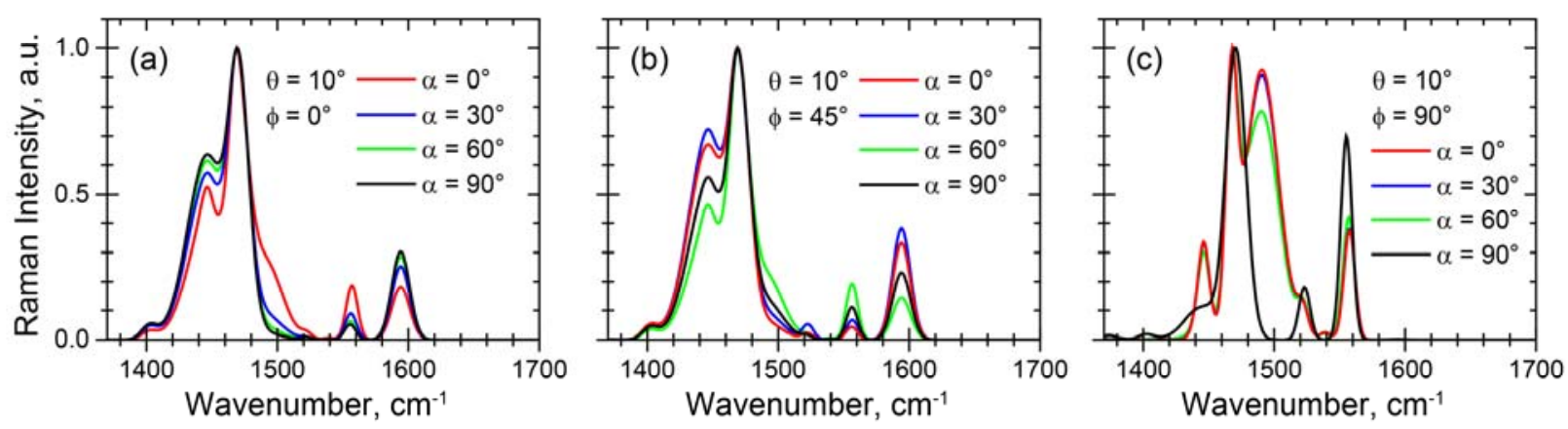

Fig. S5. Calculated anisotropy of Raman spectra assuming one molecule per a unit cell for domains with 3 various (a-c) in-plane orientations $\left(\varphi=0^{\circ}, \varphi=45^{\circ}\right.$ and $\left.\varphi=90^{\circ}\right)$ : impact of changing $\alpha$ angle. 

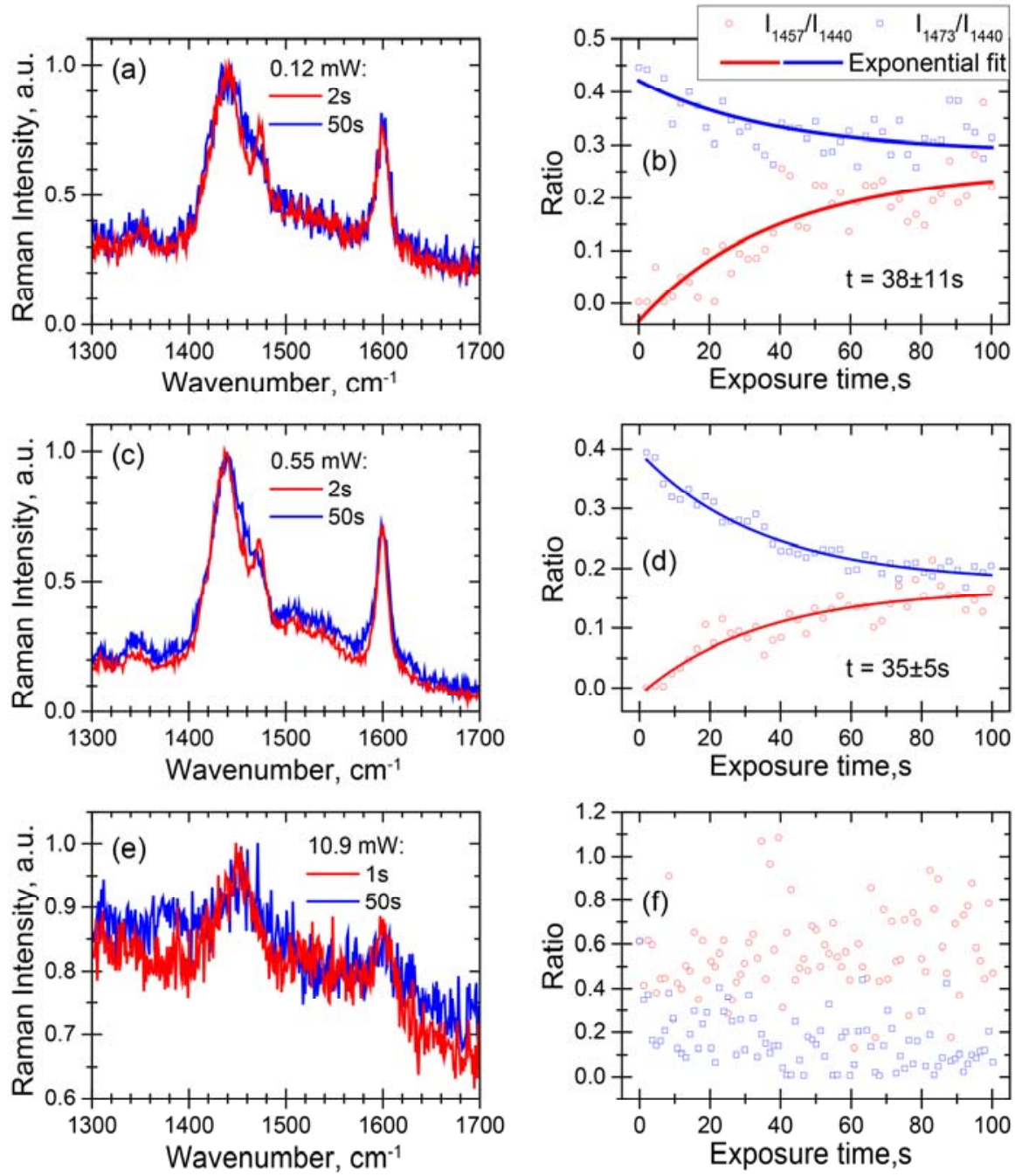

Fig. S6. Laser-induced monolayer degradation for three laser powers: $0.12 \mathrm{~mW}(\mathrm{a}, \mathrm{b}), 0.55 \mathrm{~mW}(\mathrm{c}, \mathrm{d}), 10.9$ $\mathrm{mW}(\mathrm{e}, \mathrm{f}) .(\mathrm{a}, \mathrm{c}, \mathrm{e})$ Comparison of spectra for two exposure times. (b, d, f) Normalized intensities of 1457 and $1473 \mathrm{~cm}^{-1}$ peaks dependence on exposure time. Circles are experimental data; lines are exponential fit. For all experiments except $10.9 \mathrm{~mW}$ time constants are shown as insets.

As seen in the Fig. S6. b, $\mathrm{d}$ the degradation time during the irradiation of $0.12 \mathrm{~mW}$ and $0.55 \mathrm{~mW}$ differs slightly, while using of $0.55 \mathrm{~mW}$ power allowed us to increase signal-to-noise ratio of the obtained spectra (compare Fig. S6. a, c). However, the further increasing of the laser power leads to a significant monolayer degradation. The use of $10.9 \mathrm{~mW}$ (maximum) results in a very fast degradation in the first moments of the exposure. In the Fig. S6. e very poor signal is observable only after 1 second of $10.9 \mathrm{~mW}$ irradiation. We also virtually see no characteristic degradation pattern in $I_{1457} / I_{1440}$ and $I_{1473} / I_{1440}$ dependencies on the exposure time (Fig. S6. f). The result of the degradation is observable in circular polarized light-differential interference contrast (C-DIC) optical microscopy (see pits in Fig. S7.). 


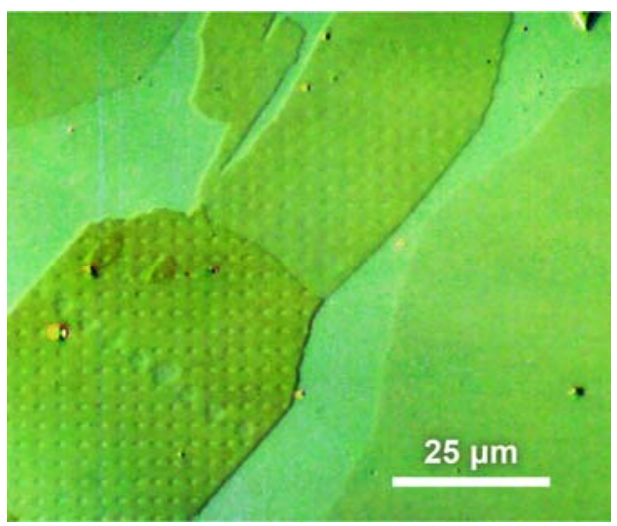

Fig. S7. C-DIC image of DH-TTPTT sample after map recording with laser power of $10.9 \mathrm{~mW}$. Accumulation time in every map pixel was 30 seconds.

\section{S4. Approximation parameters for DD-TTPTT and DH-TTPTT Raman mapping}

Table S1. Approximation parameters for DD-TTPTT SERS spectra approximation.

\begin{tabular}{|c|c|c|c|c|}
\hline $\begin{array}{c}\text { Approximation } \\
\text { curve }^{\mathrm{a}}(\#)\end{array}$ & Range $\left(\mathrm{cm}^{-1}\right)$ & Peak (\#) & Centre $\left(\mathrm{cm}^{-1}\right)$ & Width $\left(\mathrm{cm}^{-1}\right)$ \\
\hline \multirow{10}{*}{1} & \multirow{10}{*}{$\begin{array}{c}\text { from } 1270 \text { to } \\
1700\end{array}$} & 1 & 1309 & 23 \\
\hline & & 2 & 1344 & 21 \\
\hline & & 3 & 1388 & 14 \\
\hline & & 4 & 1419 & 16 \\
\hline & & 5 & 1440 & 28 \\
\hline & & 6 & 1457 & 17 \\
\hline & & 7 & 1474 & 17 \\
\hline & & 8 & 1507 & 30 \\
\hline & & 9 & 1551 & 341 \\
\hline & & 10 & 1601 & 19 \\
\hline \multirow{3}{*}{2} & \multirow{3}{*}{$\begin{array}{c}\text { from } 1250 \text { to } \\
1400\end{array}$} & 1 & 1309 & 23 \\
\hline & & 2 & 1344 & 21 \\
\hline & & 3 & 1388 & 14 \\
\hline
\end{tabular}

a Linear function was used for background approximation. Intensity of the $1388 \mathrm{~cm}^{-1}$ peak was extracted from the approximation curve 2 . Other peak intensities reported in the main text were extracted from the approximation curve \#1. 
Table S2. Approximation parameters for DH-TTPTT SERS spectra approximation.

\begin{tabular}{|c|c|c|c|c|}
\hline $\begin{array}{c}\text { Approximation } \\
\text { curve }^{\mathrm{a}}(\#)\end{array}$ & Range $\left(\mathrm{cm}^{-1}\right)$ & Peak $(\#)$ & Centre $\left(\mathrm{cm}^{-1}\right)$ & Width $\left(\mathrm{cm}^{-1}\right)$ \\
\hline \multirow{4}{*}{1} & & 1 & 1309 & 23 \\
\cline { 3 - 5 } & \multirow{3}{*}{1} & 2 & 1344 & 20 \\
\cline { 3 - 5 } & & 3 & 1388 & 14 \\
\cline { 3 - 5 } & & 5 & 1425 & 20 \\
\cline { 3 - 5 } & & 6 & 1440 & 35 \\
\cline { 3 - 5 } & & 7 & 1473 & 14 \\
\hline \multirow{2}{*}{2} & 2 & 1 & 1507 & 30 \\
\hline
\end{tabular}

aLinear function was used for background approximation. All peak intensities reported in the main text were extracted from the approximation curve \#1.

\section{S5. Additional eigenvectors}

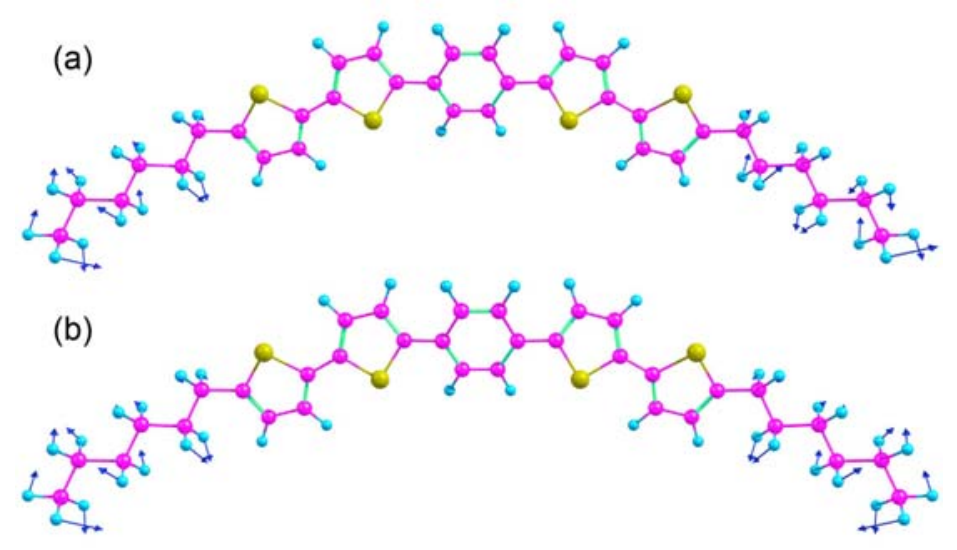

Fig. S8. Eigenvectors for 1456.86 (a) and 1456.87 (b) $\mathrm{cm}^{-1}$ calculated Raman vibrations.

\section{S6. Anisotropy of $1388 \mathrm{~cm}^{-1}$ mode}

We found $1388 \mathrm{~cm}^{-1}$ mode anisotropy to be the strongest, especially between crystalline domains and submonolayer (see Figure 3d, main text). We suppose that the $1601 \mathrm{~cm}^{-1}$ and $1457 \mathrm{~cm}^{-1}$ modes remain unchanged because of the reduction of the anisotropy in SERS discussed in the main text. We suggest that the fact that $1388 \mathrm{~cm}^{-1}$ mode is not observed in the powder can be also explained by its anisotropy.

In SERS experiments for DD-TTPTT we found $1388 \mathrm{~cm}^{-1}$ mode intensity is strong only when we measure crystalline domains, i.e. when polarization is applied almost perpendicularly to the molecule conjugated backbone (Figure 3d). While for molecules in sub-monolayer, presumably lying on the substrate this mode has almost negligible intensity. Usually, conjugated backbone vibrations Raman intensities are stronger when laser polarization is applied along the $\mathrm{S}_{0}-\mathrm{S}_{1}$ transitional dipole momentum (TDM) of the molecule. ${ }^{3}$ From the calculations, we know that $\mathrm{S}_{0}-\mathrm{S}_{1}$ TDM for TTPTT core is aligned with the molecular backbone as shown in Fig. S9. We suggest that $1388 \mathrm{~cm}^{-1}$ mode visible only in DD- substituted molecule is associated with 
non-conjugated end-groups vibration. Therefore, the powder Raman spectrum contains mostly the conjugated backbone vibrations, while for 2D crystals, relative intensities of the other modes become nonnegligible.

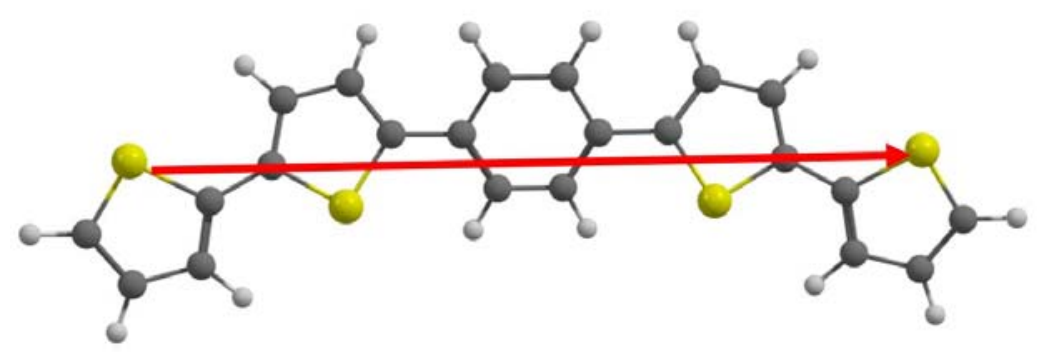

Fig. S9. TTPTT molecule and $\mathrm{S}_{0}-\mathrm{S}_{1}$ transition dipole moment direction (shown with red arrow).

\section{S7. Raman maps of DD-TTPTT and DH-TTPTT}

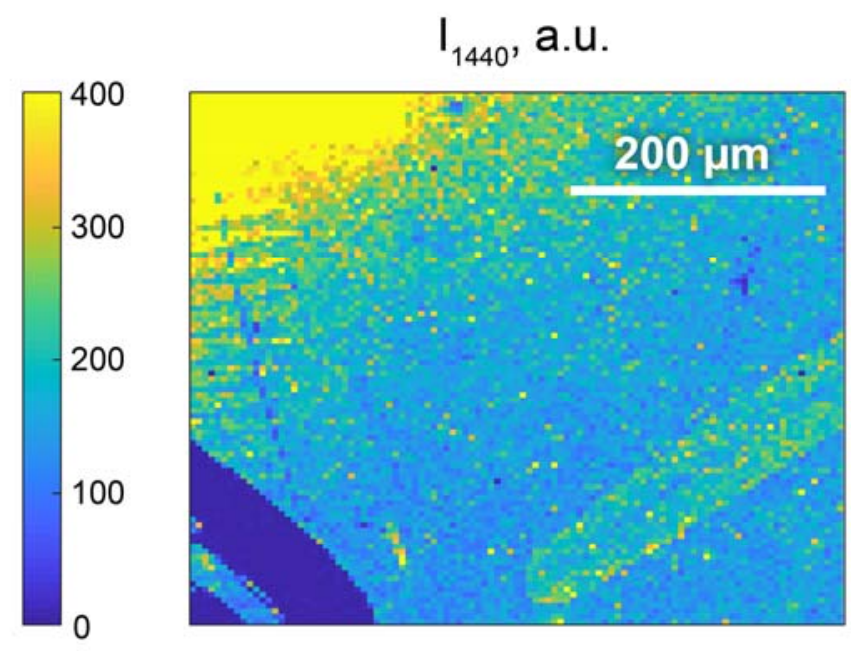

Fig. S10. DD-TTPTT map of the $1440 \mathrm{~cm}^{-1}$ peak intensity. 


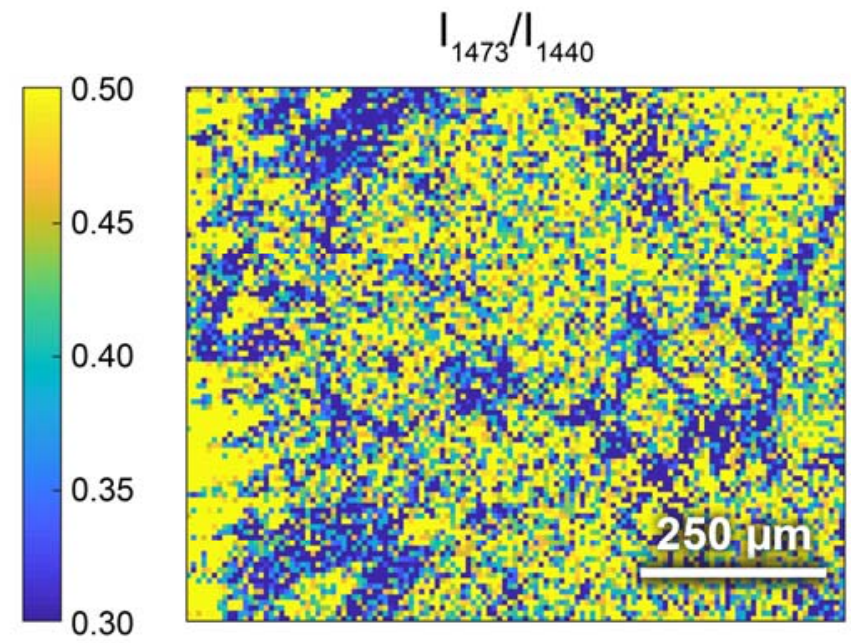

Fig. S11. DH-TTPTT map of the $1473 \mathrm{~cm}^{-1}$ peak intensity normalized to the $1440 \mathrm{~cm}^{-1}$ one.

\section{S8. DD-TTPTT AFM scans}
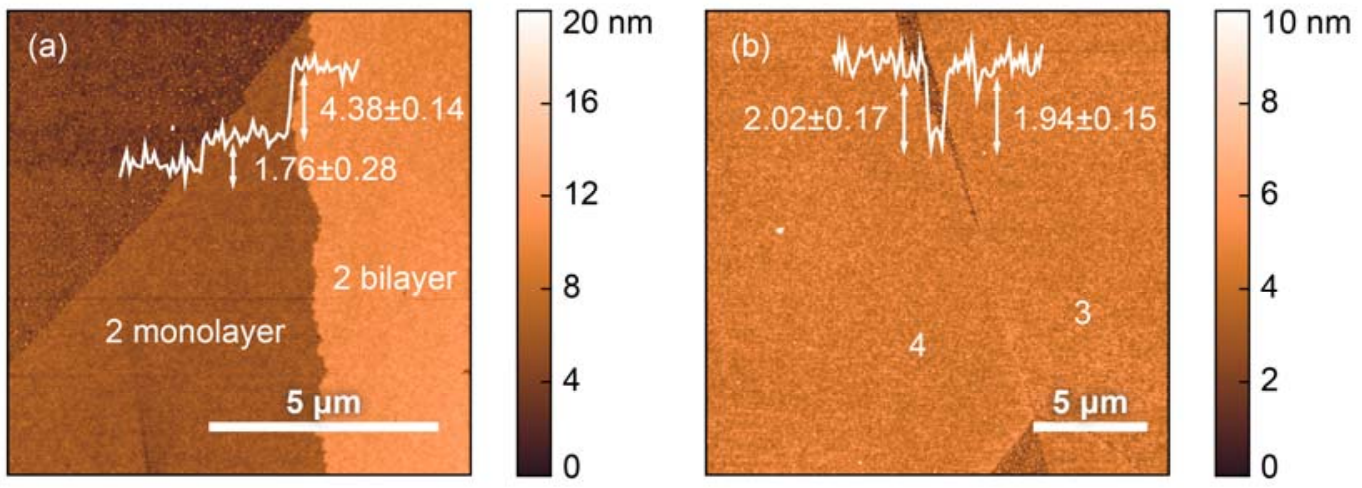

Fig. S12. AFM semi-contact scans of (a) domain \#2 including monolayer and bilayer sections, (b) domains \#3 and \#4. Insets are height profiles. 


\section{S9. Impact of different planarity on DH-TTPTT calculated Raman spectra}

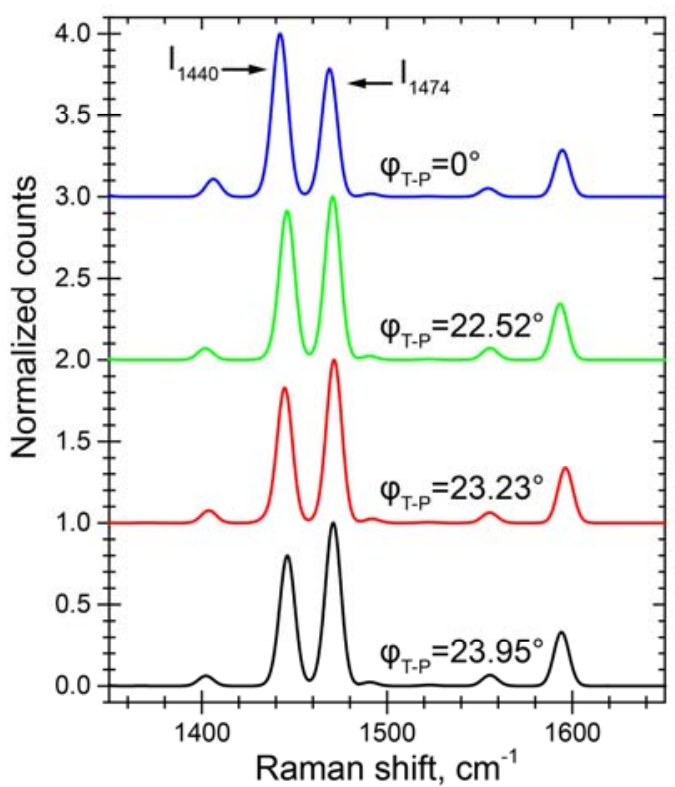

Fig. S13. Calculated Raman spectra of DH-TTPTT molecules with different angles between thiophene and phenylene rings $\varphi_{T-P}$. Peaks attributed to $1440 \mathrm{~cm}^{-1}$ and $1473 \mathrm{~cm}^{-1}$, which relative intensity was found to be strongly influenced by $\varphi_{T-P}$, are marked on the plot with arrows.

\section{References}

1. Sizov, A. S.; Anisimov, D. S.; Agina, E. V.; Borshchey, O. V.; Bakirov, A. V.; Shcherbina, M. A.; Grigorian, S.; Bruevich, V. V.; Chvalun, S. N.; Paraschuk, D. Y., et al., Easily Processable Highly Ordered Langmuir-Blodgett Films of Quaterthiophene Disiloxane Dimer for Mono Layer Organic Field-Effect Transistors. Langmuir 2014, 30, 15327-15334. 2. Bruevich, V. V.; Glushkova, A. V.; Poimanova, O. Y.; Fedorenko, R. S.; Luponosov, Y. N.; Bakirov, A. V.; Shcherbina, M. A.; Chvalun, S. N.; Sosorev, A. Y.; Grodd, L., et al., Large-Size Single-Crystal Oligothiophene-Based Monolayers for Field-Effect Transistors. ACS Applied Materials \& Interfaces 2019, 11, 6315-6324.

3. Lee Soo-Y.; Heller E. J., Time-dependent theory of Raman scattering. J. Chem. Phys. 1979, $71,4777$. 